\title{
Applying predator-prey theory to modelling immune-mediated, within-host interspecific parasite interactions
}

\author{
ANDY FENTON ${ }^{1 *}$ and SARAH E. PERKINS ${ }^{2}$ \\ ${ }^{1}$ School of Biological Sciences, University of Liverpool, Crown Street, Liverpool L69 7ZB, UK \\ ${ }^{2}$ Center for Infectious Disease Dynamics, 208 Mueller Lab, Penn State University, State College, PA, 16803, USA
}

(Received 28 Fuly 2009; revised 28 October 2009; accepted 31 October 2009; first published online 15 February 2010)

S UMMAR Y

Predator-prey models are often applied to the interactions between host immunity and parasite growth. A key component of these models is the immune system's functional response, the relationship between immune activity and parasite load. Typically, models assume a simple, linear functional response. However, based on the mechanistic interactions between parasites and immunity we argue that alternative forms are more likely, resulting in very different predictions, ranging from parasite exclusion to chronic infection. By extending this framework to consider multiple infections we show that combinations of parasites eliciting different functional responses greatly affect community stability. Indeed, some parasites may stabilize other species that would be unstable if infecting alone. Therefore hosts' immune systems may have adapted to tolerate certain parasites, rather than clear them and risk erratic parasite dynamics. We urge for more detailed empirical information relating immune activity to parasite load to enable better predictions of the dynamic consequences of immunemediated interspecific interactions within parasite communities.

Key words: parasite communities, functional response, predator-prey models, Lotka-Volterra, immune response, community stability, parasite co-infection.

\section{INTRODUCTION}

There is currently considerable interest in the potential role that within-host interactions may play in shaping the epidemiology and control of co-infecting parasites and pathogens. Most host individuals are typically infected by a community of co-infecting parasite species (e.g., Lello et al. 2004), and it is well known that strong interspecific interactions can occur between a wide range of parasite types, acting either directly, through competition for resources (such as cells) and space, or indirectly via the host's immune response (Christensen et al. 1987; Bentwich et al. 1999; Cox, 2001; Lello et al. 2004). Such interactions may potentially have important implications for parasite load and shedding rates within the host (Ebert, 1994; Otterstatter and Thomson, 2006) which, in turn, will determine transmission rates between individuals at the population level. For example, in the 2003 SARS epidemic individuals that were responsible for the majority of viral transmission (the 'super-spreaders') were those that were shedding large amounts of virus, putatively due to

* Corresponding author: School of Biological Sciences, University of Liverpool, Crown Street, Liverpool L69 7ZB, UK. Tel: +0151795 4473. Fax: +0151 795 4408. E-mail: a.fenton@liverpool.ac.uk an altered immune status arising from co-infection with other respiratory viruses (Shen et al. 2004; Bassetti et al. 2005). Similarly, co-infection of laboratory mice by microparasites and worms can result in prolonged and increased egg output of the gastrointestinal worm (Liesenfeld et al. 2004). Finally, co-infection can synergize to cause high infectioninduced host mortality, such that the co-infected individuals suffer early mortality and so are removed from the infectious population (Marshall et al. 1999).

However, although it has been known for a long time that such interactions are possible, it is only relatively recently that thoughts have moved from determining whether interspecific parasite interactions can occur, to trying to predict how they affect the dynamics, persistence and transmissibility of the parasites (Holt and Dobson, 2006; Bottomley et al. 2007; Pedersen and Fenton, 2007; Graham, 2008). Unfortunately, predicting the impact of interspecific interactions on within-host parasite dynamics is far from trivial. The inherent non-linearities associated with interspecific parasite interactions, particularly those mediated through the host's immune system, makes it extremely difficult to predict the within-host dynamics of multi-parasite communities. However, complex, non-linear, interacting dynamical systems are exactly what ecologists have been working with for decades, and it has recently been suggested that 
applying ecological concepts and techniques (e.g., Murdoch et al. 2003) to the study of within-host parasite communities may provide a way of understanding and, ultimately, predicting the dynamics of co-infecting parasite species (Bottomley et al. 2007; Pedersen and Fenton, 2007; Graham, 2008). Previous models have been developed to explore the population-level (epidemiological) consequences of multiple co-circulating parasite strains (Gupta et al. 1994; Gog and Grenfell, 2002; Ferguson et al. 2003) or species (Rohani et al. 2003; Fenton, 2008; Fenton et al. 2008), often involving a phenomenological representation of the degree of cross-immunity between parasites. To date, however, there have been few attempts to develop mechanistic models examining immune-mediated within-host interactions between co-infecting parasite species.

In this paper we present a suite of models, based on existing ecological frameworks, and explore their suitability for predicting the within-host dynamics of multiple co-infecting parasites. In particular, we focus on immune-mediated interactions, rather than direct resource competition between parasites, because the additional dynamical complexity associated with multi-species parasite communities interacting with, and via, the host's immune system makes it essential to develop an appropriate theoretical framework to aid the understanding of such highly nonlinear systems. Using a suite of theoretical models we consider how different configurations of parasite communities and their interaction with the host's immune response lead to very different outcomes, in terms of disease persistence. Overall, we show that a variety of outcomes are possible, depending on the configuration of the community. Where possible we annotate this paper with empirical examples. However, we urge for more in vivo work that quantifies the specific relationship between the magnitude of the host's immune response and the rate of pathogen clearance, across a range of realistic parasite levels.

\section{DEVELOPMENT OF THE MODELS}

\section{Existing single-species models of immune-mediated parasite dynamics}

An obvious starting point to develop models of multiple interacting parasite species is to use existing models of single-species dynamics. There has been a considerable body of theory developed to elucidate the within-host dynamics of a single infecting pathogen and its interaction with the host's immune response (Antia et al. 1996; Antia and Lipsitch, 1997; Nowak and May, 2000; Pilyugin and Antia, 2000; Antia and Koella, 2004). These single-species models have borrowed heavily from existing ecological theory and typically assume a 'predatorprey'-type relationship where the immune system acts like a predator, attacking the invading parasite (the prey). This analogy provides a reasonable first approximation of parasite-immune system dynamics (but see Wodarz, 2006), and allows use of the rich body of predator-prey theory. Central to predatorprey theory is the functional response, which describes how the predator's feeding rate changes in response to prey density. Importantly, different types of predators and prey have different forms of this functional response, affecting their population dynamics and stability of the interaction. By extension to parasite-immune system models, the shape of the immune system's functional response (i.e. the relationship between parasite load and the rate at which it is cleared by the immune system) will vary according to the nature of the invading parasite, with important implications for the ability of the host to eliminate the infection and the parasite's transmission dynamics at the population level. Therefore, determining the functional response for a specific parasite-immunity interaction can provide invaluable information for the design of long-term disease control strategies. However, existing models of the within-host parasite-immune system interaction typically assume a very simple, restrictive form of the functional response (see below), meaning many of the predictions ignore a large range of potential outcomes. Clearly this needs to be addressed before we can extend these models to consider a community of co-infecting parasites and their potentially complex network of immune-mediated interactions.

\section{Applying predator-prey theory to parasite-immune system interactions: the role of the functional response}

First, as a baseline, we describe the standard approach for modelling single species within-host dynamics, and consider the value of broadening it to account for more complex interactions with the host's immune response. In the subsequent section we extend this framework further to explore the consequences of incorporating multiple, interacting parasite species. At this stage we only consider the simplest representation of the parasite-immune system interaction, using standard, deterministic models and ignore more complex features such as explicit time delays in the mounting of an effective immune response, although more complex models may easily be derived from this baseline framework (e.g., Buric et al. 2001; Fenton et al. 2006). Note that, since many of these models are standard from the ecological literature, we only present the key results in this paper, and leave mathematical details of the stability analyses in the Online Appendix (Online version only).

The most common application of predator-prey theory to parasite-host immune system dynamics (e.g., Nowak and May, 2000) makes use of the 

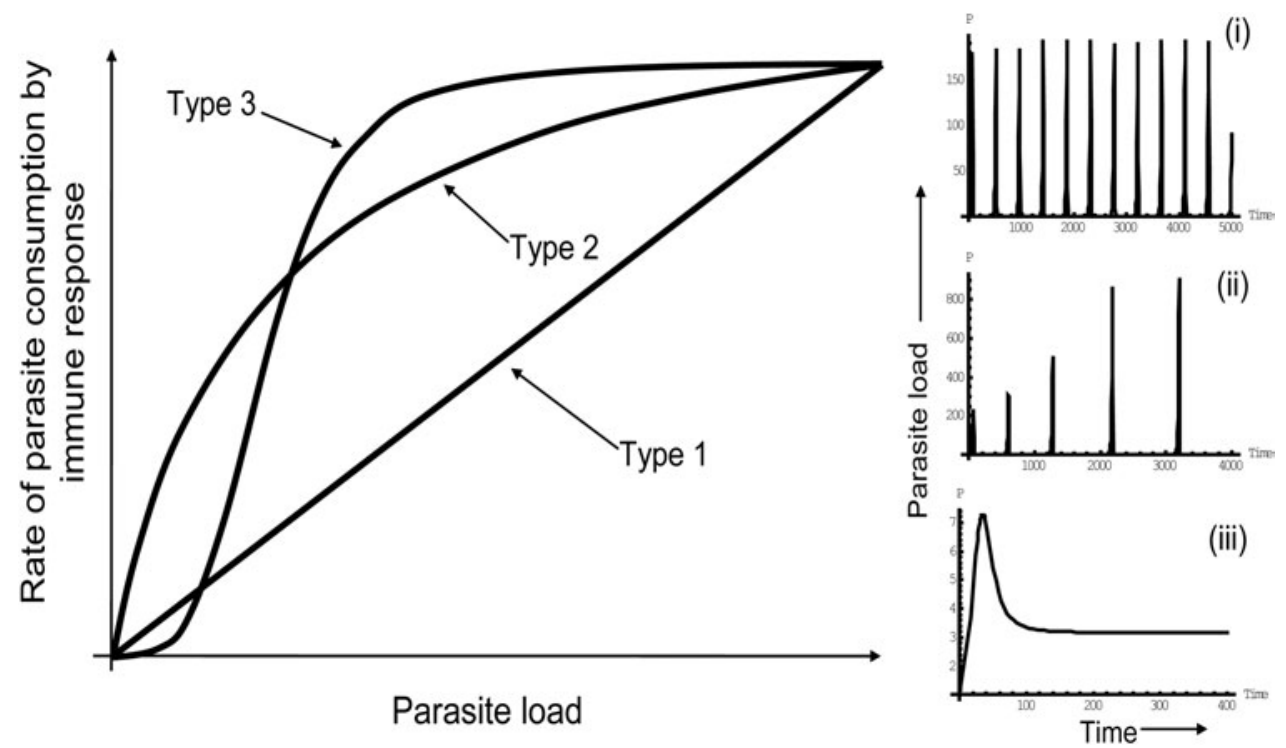

Fig. 1. Relationship between parasite density and consumption of the parasite due to the immune response [the immune system's functional response, $f(P)$ ] for Type 1, Type 2 and Type 3 functional responses. The inset graphs show time series of the dynamics of a microparasite population (Model 1) under (i) a Type 1, (ii) Type 2 and (iii) Type 3 functional response.

Lotka-Volterra model (Model 1):

$$
\begin{aligned}
& \frac{d P}{d t}=r P-I f(P) \\
& \frac{d I}{d t}=e f(P) I-\delta I
\end{aligned}
$$

Model 1

Definitions and baseline values for all parameters are provided in Table A1 in the Online Appendix. In terms of the within-host parasite dynamics, $P$ represents the size of the parasite load (the 'prey' population), which grows at per capita rate $r$ and is consumed by the active components (e.g., macrophages or antibodies) of the immune system, $I$ (the 'predator' population) according to the functional response $f(P)$. Immune proliferation is stimulated through contact with parasitic antigens at rate $e$, and the active component of the immune system decays at rate $\delta$. One important point is that model formulations like Model 1 most accurately reflect infections by microparasites such as viruses and bacteria, which replicate within the host. When the infection is due to a macroparasite (e.g., a parasitic helminth) that does not replicate within the host the dynamics are more accurately modelled by:

$$
\begin{aligned}
& \frac{d P}{d t}=\Gamma-I f(P) \\
& \frac{d I}{d t}=e f(P) I-\delta I
\end{aligned}
$$

Model 2

where $\Gamma$ is the rate at which the host acquires parasitic infection. As we show below, the within-host dynamics greatly depend on which formulation is used although, in either case, the functional response is key to the dynamics of the system. Typically this is described using one of three functional forms (Fig. 1):

Type 1 Functional Response: $f(P)=\beta P$. This is the simplest scenario to analyse and the one adopted by the majority of models of within-host parasite dynamics (e.g. Antia et al. 1996; Nowak and May, 2000), whereby a constant proportion of the parasite population is consumed by the immune response at rate $\beta$. Type 1 responses may arise through the presence of long-lived immune memory, which reduces the delay associated with affinity maturation of the specific immune response and the production of antibodies by B-cells, allowing for a rapid and highly specific response to a re-invading parasite, even at low parasite loads (Zinkernagel, 1996).

The dynamics resulting from a Type 1 functional response are typically neutrally stable (Appendix A1, Online version only), potentially resulting in highamplitude oscillations (Fig. 1, inset i). These may be characterized by an initial rapid expansion of parasitaemia followed by elimination of the parasite as it passes through the very low trough in abundance. However, if the invading parasite is a macroparasite (Model 2), the parasite is always regulated to a stable equilibrium by the immune system. Therefore, although chronic microparasite infection is very unlikely to result under a Type 1 functional response, it is highly likely for a macroparasite.

Type 2 Functional Response: $f(P)=\frac{\beta P}{1+h \beta P}$. Here a decreasing proportion of the parasite population is consumed as parasite load increases. In predatorprey systems this is typically due to predator saturation at high prey densities, where predators are 
limited by the handling time $(h)$ taken to process each prey item. For within-host dynamics Type 2 responses imply that the rate of immune action saturates with increased parasite load. Type 2 responses are rarely considered in models of the immune system but they may occur for cell-mediated responses, where a significant handling time is required for macrophages and phagocytic cells to deal with an infected cell or extracellular parasite (Pilyugin and Antia, 2000). Models incorporating Type 2 functional responses tend to be unstable (Appendix A1, Online version only), resulting in divergent oscillations (inset ii, Fig. 1), ultimately leading to the parasite either being eliminated as it passes through a trough of the cycle, or to the parasite or immune response building up to dangerously high levels, as it passes through a peak. As with Type 1 responses, chronic microparasite infections are unlikely for Type 2 responses, but they are possible for a macroparasites (Model 2), providing immune stimulation is sufficiently high (i.e., providing $e>\delta h$; Table A2).

Type 3 Functional Response: $f(P)=\frac{\beta P^{2}}{1+h \beta P^{2}}$. Here, parasite clearance follows a sigmoidal pattern such that at low densities the clearance rate is low, accelerating only when the parasite load becomes high. In predator-prey systems this occurs when the predator is inefficient at foraging for rare prey, and is typically generated when the consumer switches between prey species (i.e., it develops a 'search image' for the more common prey types; Holt, 1983). In terms of the host's immune response, this is analogous to the period of clonal expansion and affinity maturation, as the specific immune response develops towards the invading parasite. This is therefore likely for primary infections caused by novel parasites invading hosts with no prior immune memory. Extended delays in the build-up of affinity may be particularly pronounced for parasites which undergo antigenic shifts to keep ahead of the immune system (e.g., Schistosoma spp.). Type 3 functional responses can produce stable dynamics (inset iii, Fig. 1). Specifically, stability occurs if $e>2 \delta h$ for a microparasitic infection (Model 1; Table A2) and $e>\delta h$ for a macroparasitic infection (Model 2; Table A2). If these criteria are reversed, the parasite escapes regulation and increases to dangerously high levels. However, if the host develops long-lived immune memory such that it does not undergo a prolonged period of clonal expansion following re-infection by the same parasite species or strain (depending on the degree of cross-reactivity), a Type 3 primary response may change to a Type 1 secondary response upon re-infection, leading to rapid expulsion of the parasite.

In general, stability of all models, where possible, is determined by the rate of stimulation of the immune response $(e)$, the rate of immune decay $(\delta)$ and the 'handling time' $(h)$ required for the immune system to deal with an infected cell or invading parasite (Table A2). Furthermore, the equilibrium pathogen load $\left(P^{*}\right)$ is determined by a combination of these three parameters and the rate at which the immune system consumes the parasite $(\beta)$. Hence, efforts should be directed towards obtaining empirical measurements of these parameters where possible from laboratory or experimental systems. However, the rate of microparasite replication $(r)$, or macroparasite invasion $(\Gamma)$ have little dynamical impact and so these parameters are not so important to obtain estimates for in single species infections.

\section{EXTENDING THE ANALOGY: CO-INFECTIONS} AS PREDATOR-PREY COMMUNITIES

As with the use of predator-prey theory to model single parasite species interacting with host immunity, within-host parasite communities, and their associated immune responses, may be considered similar to ecological communities comprising a number of free-living species (Pedersen and Fenton, 2007). In this case, the different components of the host's immune system are akin to a suite of predators consuming a number of prey species (the withinhost parasite community). Extending predator-prey models to include alternative predator or prey species lead to considerably different dynamics from those seen for the component species in isolation (Holt, 1983; Holt and Lawton, 1994; Bonsall and Hassell, 1997; Murdoch et al. 2003). Clearly, the same is likely to be true for host-multiparasite interactions; ignoring the effects of co-infecting parasite species could give a misleading impression of the dynamics of the system.

As with ecological communities, parasite communities may take a number of possible forms, depending on the nature of the component species and their mechanisms of interaction. Here we focus on interactions that are mediated by the host's immune response rather than, for example, through direct competition for a common resource (e.g., competition between helminths that induce anaemia and microparasites that depend on red blood cells, such as Plasmodium sp.; Graham, 2008). Clearly, such immune-mediated interactions will depend on the antigenic nature of the invading parasites, their levels of cross-immunity and the host's allocation of resources to the different components of the immune system that target each parasite. As a generalization, microparasites (e.g. viruses and bacteria) result in the production of different cytokines from those of macroparasitic infections (e.g. parasitic helminths). Specific cytokines are involved in cross-regulation of others, thus potentially altering the immune response to a co-infecting parasite (Callard and Yates, 2005). Therefore, depending on the immunemeditated interaction between the parasites one 
(a)

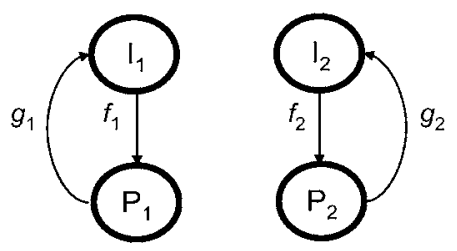

(b)
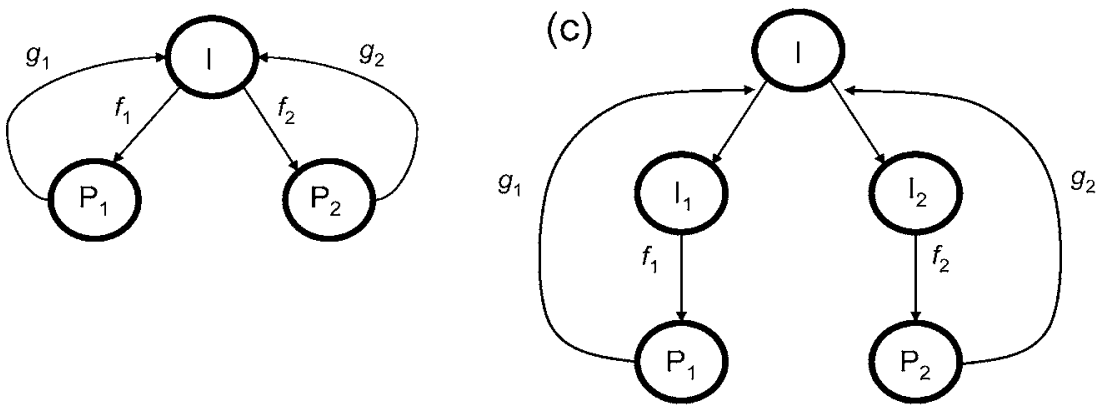

Fig. 2. Community modules of within-host co-infection scenarios. In each case the host's immune response to parasite $P$ is denoted by $I$. The functional response to parasite species $i$ is denoted by $f_{i}$, whilst the numerical response of the host's immune system due to the presence of species $i$ is $g_{i}$. (a) Non-interacting co-infections, (b) co-infections with cross-immunity, where the host is simultaneously co-infected by two different parasite types which interact via a shared immune response and (c) co-infections with antagonistic immune responses, where the host is simultaneously co-infected by two different parasite types but each parasite initiates a different, antagonistic response of the immune system.

could indirectly affect another, by increasing or decreasing the efficacy of the immune response towards it. We propose that the majority of coinfection scenarios that involve the host's immune system can be characterized by one of three different community configurations (note that, for simplicity, we concentrate on communities involving only two parasite species, although the main principles can be generalized to communities involving more species; Fig. 2):

Non-interacting co-infections (Fig. 2a). This scenario assumes that concurrent infections occur independently from each other, and that the host mounts independent immune responses to each parasite (i.e. a non-systemic, localized immune response that results in no within-host competition, toxin production or cross-regulation of immune parameters). Following the predator-prey analogy these immune responses may be thought of as specialist predators each consuming an individual prey species. As a result, the dynamics of the full parasite community are simply determined by the separate dynamics of each parasite-immune response pair. Hence, parasites that elicit Type 1 or Type 2 functional responses will remain unstable, whereas parasites that elicit Type 3 functional responses may be stable, regardless of the presence of the other parasite species. This scenario is most likely for low-intensity infections involving antigenically unrelated parasite species with no cross-immunity in well-resourced hosts, or for parasites occupying separate physical locations within the host, with no direct competition for resources. In reality, however, there may be some degree of cross-immunity between parasite species, resulting in community configuration 2 (see below) or hosts may be energetically compromised, resulting in community configuration 3 (see below).

Co-infections with cross-immunity (Fig. 2b). When antigenically related parasites infect the same host there may be immune-mediated interactions, where the response raised against one parasite affects the growth of the other. Here, the degree of crossimmunity between the parasites is crucial; this crossimmunity is not necessarily due to phylogenetic relatedness between the parasites, but the degree of antigenic similarity, allowing antibodies and T-cells raised against one parasite to recognize antigens on the other. It should be noted that parasites may interact in this way even if they do not infect the host at the same time, providing there is some long-lived immune memory remaining from the initial infection (Koelle et al. 2005).

This scenario closely resembles predator-prey communities where a single generalist predator feeds on several prey species. Considerable theoretical work has been undertaken on this configuration in community ecology, showing that even if the two prey species do not compete directly for resources, the presence of one may still have a negative effect on the other, mediated by the generalist predator 
a)

(i) Both microparasites

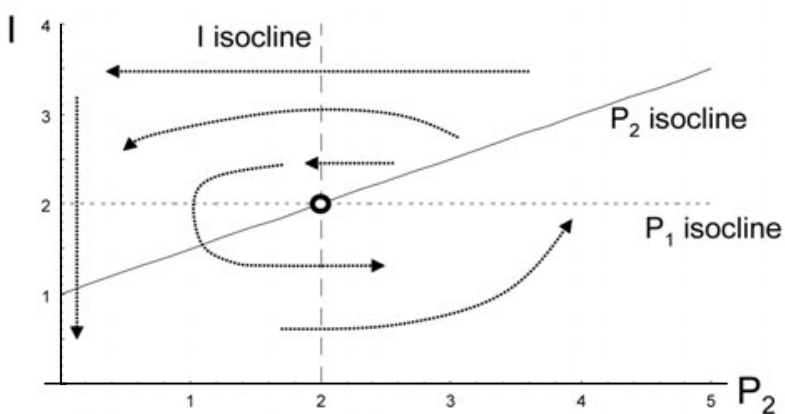

b)

(i) Both microparasites

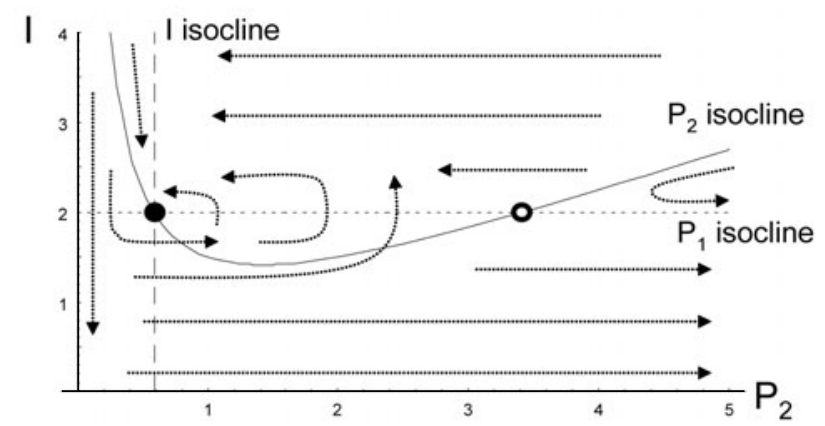

(ii) Both macroparasites

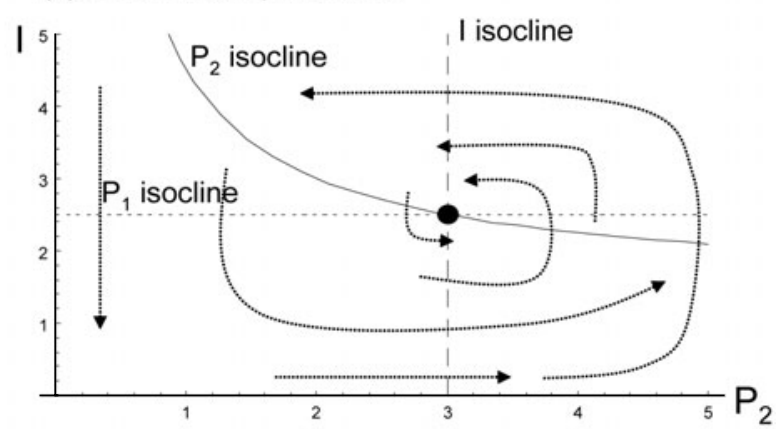

(ii) Both macroparasites

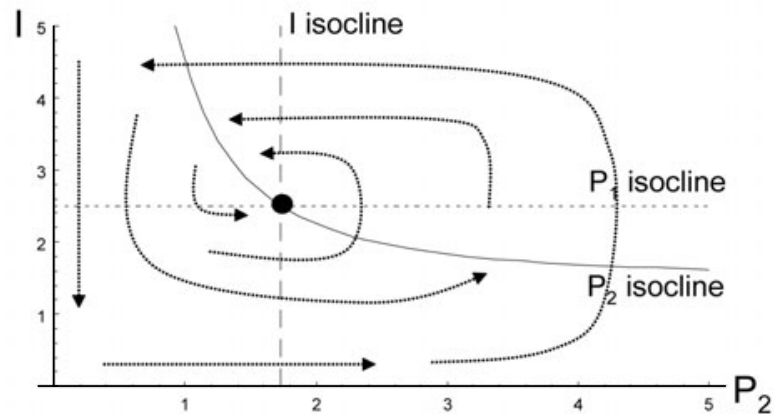

Fig. 3. Phase plots in $P_{2}-I$ state space for two co-infecting microparasite species (Figs 3ai and 3bi) and two co-infecting macroparasite species (Figs 3aii and 3bii) which interact via a shared immune response (Models 1 and 2). (a) Parasite $P_{2}$ elicits a Type 2 functional response and parasite $P_{1}$ elicits a Type 1 functional response and (b) parasite $P_{2}$ elicits a Type 3 functional response and $P_{1}$ elicits a Type 1 functional response. The solid line is the $P_{2}$ isocline, the dashed line the $I$ isocline and the dotted line the $P_{1}$ isocline. The arrows represent the direction of dynamical flow within the state space.

(a phenomenon termed apparent competition; Holt, 1977; Holt and Lawton, 1994). These concepts can be applied to co-infecting parasite species which interact via the host's immune system through antigenic cross-reactivity, such that infection by one parasite may have a detrimental impact on the abundance of another, via the host's immune response. However, what is not often appreciated is that such cross-immunity may also allow one species to have a positive effect on the stability of another species, potentially facilitating its persistence when it would otherwise either be eliminated. We illustrate this using isocline analysis, where the Zero Net Growth Isoclines (ZNGIs) represent curves in phase space depicting species combinations where the growth rate of each species is zero; the intersections of these lines represent equilibria of the system, which may be stable or unstable. The two-parasiteimmune system model can be described by:

$$
\begin{aligned}
& \frac{d P_{1}}{d t}=r_{1} P_{1}-I f_{1}\left(P_{1}\right) \\
& \frac{d P_{2}}{d t}=r_{2} P_{2}-I f_{2}\left(P_{2}\right) \\
& \frac{d I}{d t}=I\left[e_{1} f_{1}\left(P_{1}\right)+e_{2} f_{2}\left(P_{2}\right)-\delta\right]
\end{aligned}
$$

where the $P_{\mathrm{i}}$ are the within-host densities of parasite species or strain $i, I$ is a measure of the magnitude of the shared immune response and other parameters are as defined for Model 1. Note that the functional response of the immune system may differ for the different parasite species (as denoted by the $f_{i}\left(P_{i}\right)$ terms). However, for simplicity, we assume that since the shared immune response is described by a single term $(I)$, its decay rate $(\delta)$ is constant, and independent of the nature of the organisms eliciting it. Based on this model framework, the three ZNGIs are obtained by setting the above equations equal to 0 and plotting them in the 3-dimensional space $P_{1}-P_{2}-I$ (although, for ease of representation we show a slice through this cube, plotting the isoclines in $P_{2}-I$ phase space; Fig. 3 ).

If both microparasite species elicit a Type 1 functional response (i.e. $f_{i}\left(P_{\mathrm{i}}\right)=\beta_{\mathrm{i}} P_{\mathrm{i}}$ for $\left.i=1,2\right)$, the $Z N G I s$ for the two parasite species never cross, regardless of the relative densities of the parasites, so coexistence of both parasite species is impossible (see Appendix A2.1 for mathematical details, Online version only). Furthermore, if both parasites are microparasites (Model 1) and one of them elicits a Type 2 response and the other has either a Type 1 or Type 2 response, the system is inherently unstable 
and parasite coexistence is again impossible, as the system undergoes divergent oscillations (Fig. 3ai). However, if both parasites are macroparasites (Model 2), then stability can occur regardless of the shape of either functional response (Fig 3aii; Appendix A2.2); hence, parasite communities comprising macroparasites with cross-immunity may be expected to always exhibit stable, chronic infections, as is frequently observed (Ben-Smith et al. 1999).

Importantly, if both parasite species are microparasites and either one of them elicits a Type 3 functional response, stability of the entire community is possible, even if the other species elicits a Type 1 or Type 2 functional response (Fig. 3bi). Specifically, stable coexistence occurs if $\beta_{2}>\frac{4 \beta_{1}^{2} h_{2} r_{2}^{2}}{r_{1}^{2}}$ (Appendix A2.1). Hence, if the rate of regulation of the parasite species eliciting the Type 3 functional response $\left(\beta_{2}\right)$ is high enough to prevent it from escaping the immune response then it can have a sufficiently stabilizing effect to allow chronic infections of parasites that would otherwise go extinct. An important point here is that the stable coexistence is determined by the rates of microparasite replication $(r)$ and the rate of parasite consumption by the immune response; parameters that did not appear important in the corresponding single-species models above (where the rates of stimulation and decay of the immune response, $e$ and $\delta$, were important). Hence, the key parameters, and hence the focus of empirical investigations, differ under co-infection from those under single infection.

Finally, it should be noted that combinations of high infection burdens of the parasite eliciting the Type 3 response and low initial levels of immunity may be unstable (Fig. 3bi), allowing the parasite to escape the immune response and undergo rapid growth. This possibility is less likely if both parasite species are macroparasites (Fig. 3bii), again emphasizing why macroparasites may produce chronic infections even in the presence of other, co-infecting parasites.

Co-infections with antagonistic immune responses (Fig. 2c). Finally, we consider the case where antigenically dissimilar parasites interact via the host's immune system. This can occur if the ability of the host to mount an effective response to one parasite is reduced due to the need to mount a response against a second co-infecting parasite species. A classic, albeit simplified, example of this is the Th1-Th2 dichotomy, where microparasites tend to promote a Th1 immune response and macroparasites tend to induce a counter-regulating Th2 response (Abbas et al. 1996). Note that this dichotomy is by no means a universal law (for example some bacteria may induce a Th2 response); all that is sufficient at this stage is to acknowledge that some parasites induce one response, and other parasites induce the other, leading to a potential interaction between the two.
Typically, these two arms of the immune response are antagonistic, resulting in potential indirect interactions among the parasite community. For example, primary infection with the microparasite Toxoplama gondii inhibits the development of Th2 immune responses against the helminth Nippostrongylus brasilensis, allowing prolonged growth and egg output (Liesenfeld et al. 2004). Conversely, helminth infection with Fasciola hepatica suppresses the Th1 response to Bordetella pertussis, resulting in increased bacterial growth during co-infection (Brady et al. 1999).

Following the predator-prey analogy, this scenario may be thought of as a multi-predator-multi-prey community, where each arm of the immune response represents a specialist predator for the given prey (parasite) type, but the predators are limited by some other extrinsic process. A candidate model for this scenario is :

$$
\begin{aligned}
\frac{d P_{1}}{d t} & =r_{1} P_{1}-I_{1} f_{1}\left(P_{1}\right) \\
\frac{d P_{2}}{d t} & =r_{2} P_{2}-I_{2} f_{2}\left(P_{2}\right) \\
\frac{d I_{1}}{d t} & =I_{1}\left[e_{1} P_{1}\left(1-\frac{I_{1}+I_{2}}{K}\right)-\delta_{1}\right] \\
\frac{d I_{2}}{d t} & =I_{2}\left[e_{2} P_{2}\left(1-\frac{I_{1}+I_{2}}{K}\right)-\delta_{2}\right]
\end{aligned}
$$

Model 4

where $I_{1}$ and $I_{2}$ represent the specific components of the immune response (e.g. each arm of the Th1-Th2 dichotomy) that respond to parasite types 1 and 2 respectively. In community ecology, $K$ represents a common carrying capacity for the predator species, possibly arising through competition for space. A direct analogy with the immune system is less clear, but $K$ may be thought of as the population of undifferentiated T-cells that either become Th1 or Th2 cells, depending on the relative infection pressures of micro- or macroparasites.

The limiting nature of the host's immune response has important consequences for the dynamics of the system. To illustrate this we firstly consider single infections by parasite species 1 only (i.e. $P_{2}=I_{2}=0$ ). For a Type 1 functional response stability is given by the condition $r<\beta K$ (Fig. 4; see Appendix A3 for mathematical details, Online version only). If this condition is unfulfilled, the parasite can overwhelm the limited immune response. As with the previous models without limited immunity, Type 2 functional responses are destabilizing whereas Type 3 responses can be stabilizing (Fig. 4). However, unlike the previous model, the presence of this upper limit to the immune response means that stability with a Type 2 response is possible, providing the rate of parasite clearance by the immune system $(\beta)$ is sufficiently large (i.e., $\beta>r \sqrt{e} / K(\sqrt{e}-2 \sqrt{r K h \delta})$; Appendix A3). If this condition is not fulfilled then 


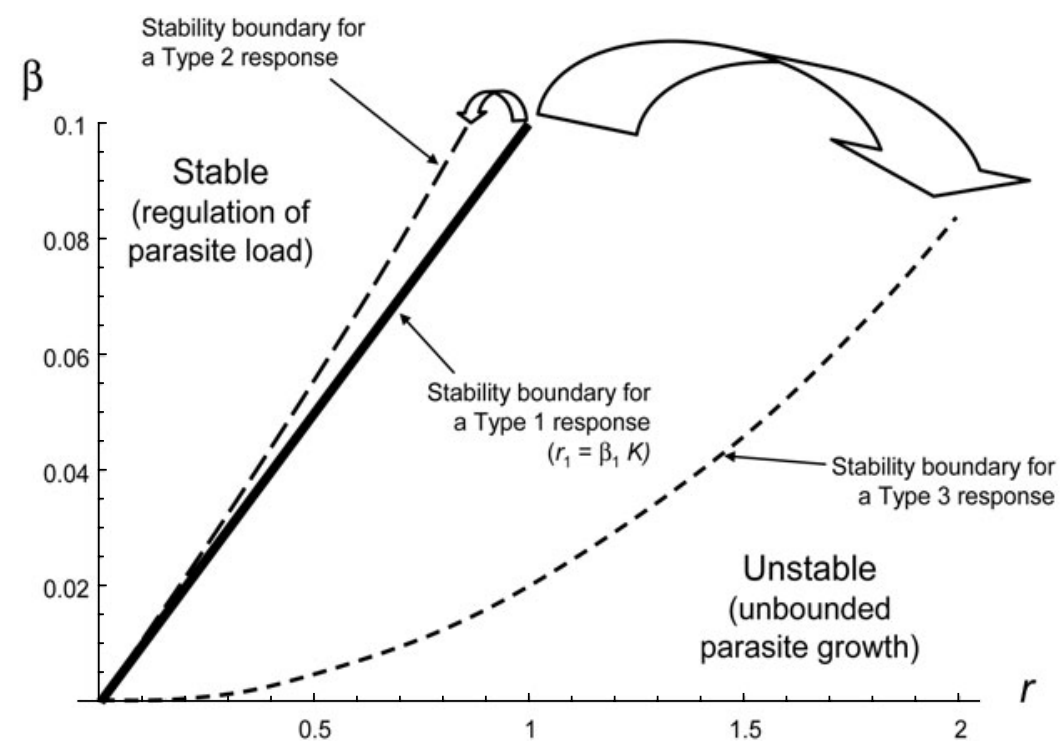

Fig. 4. $r_{1}-\beta_{1}$ parameter space for a single parasite species infecting a host with a limited immune response. The solid line shows the boundary separating stable from unstable (exponential growth) dynamics of the parasite assuming the immune system exhibits a Type 1 functional response. The dashed and dotted lines show how this boundary moves for a Type 2 and Type 3 functional response respectively.

the parasite escapes regulation and grows exponentially.

As before, the introduction of a second parasite species into the community can affect the stability of the other species. We illustrate this for a community where both parasites elicit Type 1 functional responses with their respective components of the immune system. While for single species infections stability occurs if $K>r / \beta$, in the case of multi-species infections stability only occurs if (Appendix A3):

$K>\frac{r_{1}}{\beta_{1}}+\frac{r_{2}}{\beta_{2}}$ criterion 1

Hence, unlike that observed in section 2 above, the presence of a second parasite species reduces stability compared to that of a single-species infection (Fig. 5). Therefore, stability of the parasite community is determined by the additive net reproductive rates of the component species; if criterion 1 is broken, the species with the greatest $r_{\mathrm{i}} / \beta_{\mathrm{i}}$ escapes regulation by the immune system and grows exponentially.

\section{DISCUSSION}

There is a growing interest in the role that interspecific interactions between co-infecting parasites play in determining the epidemiology of the component species, the efficacy of different control strategies and host health (see Pedersen and Fenton, 2007 for a review). However, there has been little theoretical work aimed at understanding how interactions between the component species of the parasite community affect their within-host dynamics. These within-host interactions are crucial for determining the transmissibility of each parasite species and whether the parasites are eliminated by the host, achieve long-term chronic infection, or escape the host's immune response, potentially resulting in death of the host.

To describe possible configurations between two co-infecting parasite species and the host's immune response we modified approaches currently used in free-living community ecology. Our parasite community configurations resemble those of Holt and Dobson (2006), but differ in the important fact that ours specifically describe within-host community configurations, rather than configurations across a range of host and parasite community scales. Therefore the dynamical consequences arising from our configurations may be directly compared, allowing a clear understanding of the processes facilitating parasite elimination or proliferation. Our analyses show that both the type of parasite (i.e., whether it is a microparasite that multiplies directly within the host or a macroparasite which does not) and the nature of any immune-mediated interaction between co-infecting parasites (i.e., whether there is crossimmunity or an antagonistic immune response) are important for determining the stability of the parasite community. However, the results are crucially dependent on one of the most important, but overlooked, features of within-host parasite communities: the immune system's functional response towards each parasite species. This response describes how the rate of parasite clearance varies with parasite infection load, and is determined by a range of factors including whether the host has experienced that parasite or its antigens before (i.e., whether there 


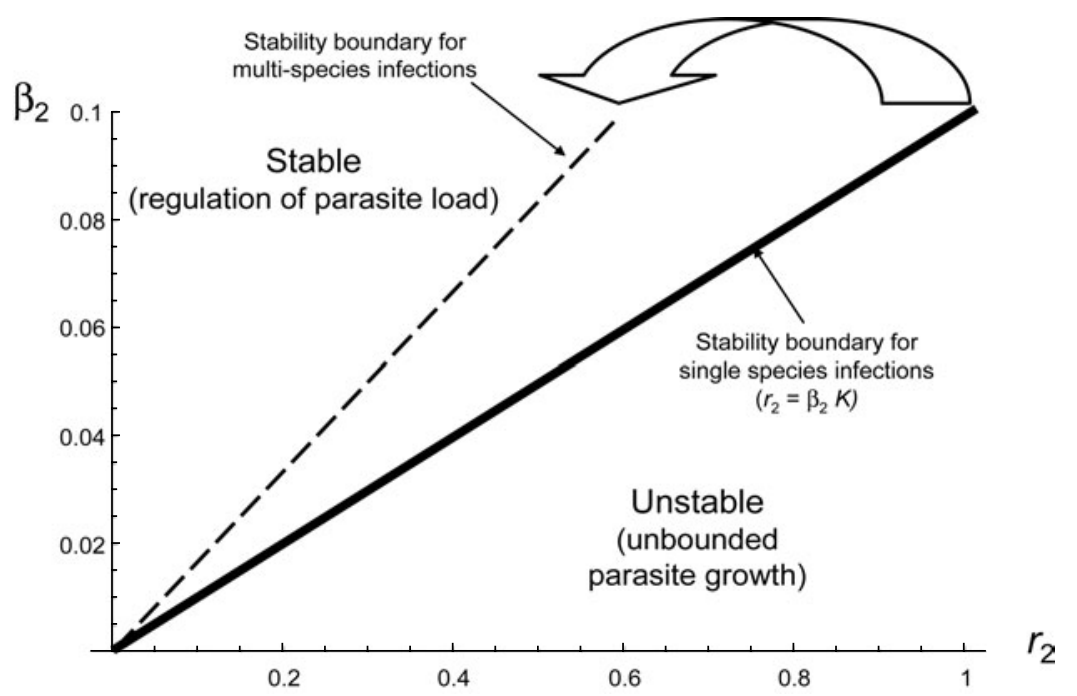

Fig. 5. $r_{2}-\beta_{2}$ parameter space for two parasite species interacting via an antagonistic immune response (Model 3). The solid line shows the boundary separating stable from unstable dynamics of a single infecting parasite and the dashed line shows how this boundary moves in the presence of a second parasite species eliciting a Type 1 functional response.

is immune memory), the time taken to neutralize infected cells or extracellular parasites, and the duration of affinity maturation. As with free-living community ecology, different forms of this functional response result in very different outcomes in terms of the stability of the infection and the ability of the host to eliminate the parasite.

Of crucial importance is the recognition that the interaction of co-infecting parasites which elicit different functional responses can have important implications for the dynamics and stability of the parasite community as a whole. There has been considerable work in community ecology aimed at understanding the relationship between community complexity and stability, showing that complex communities are not more stable than simple ones, unless the communities have a certain configuration (May, 1974; Sole and Montoya, 2001; Borer et al. 2002). As we show here, a single parasite eliciting a Type 3 functional response can stabilize the dynamics of other, co-infecting parasites that would otherwise have unstable within-host dynamics. In this sense it may be beneficial for the host to tolerate certain parasitic infections which result in stable, controlled dynamics of the entire parasite community, rather than attempt to clear them, potentially resulting in highly erratic and unpredictable parasite dynamics. Indeed, previous empirical work suggests that tolerance of parasites is an evolutionary strategy by which hosts avoid immunopathology (Read et al. 2008). These predictions would not be made if we simply extended current within-host parasite models that assume a Type 1 functional response.

Due to the importance of the immune system's functional response to the dynamics of the within-host parasite community, we believe more work should be devoted to quantifying these relationships. Despite many studies that have quantified micro- or macroparasite doses and various immune measures (e.g., antibody or B-cell levels) throughout the course of an infection (Nowak and May, 2000; Perelson, 2002), there is a lack of data that shed light on the form of the immune system's functional response. For this we need to quantify how the rate at which parasites are attacked by the immune system varies with parasite load. This has previously been addressed in insects, showing that the immune system's functional response is very different from the simple formulations used in current models (Otterstatter and Thomson, 2006). Clearly there are logistical difficulties associated with quantifying such a process in vertebrate hosts. There may be two ways to achieve this that correspond to the ways in which predator functional responses are quantified in the ecological literature. Firstly, data could be obtained directly by measuring the proportional reduction of parasite or pathogen load across a range of realistic inocula levels within a specific time frame. This is equivalent to classical lab experiments (e.g., Gause, 1935, 1936) involving replicated, controlled incubations of predators and prey populations, and are the most direct method of determining the form of the functional response. Secondly, a variety of models with different functional responses could be fitted to longitudinal time series of parasite load and relevant immune levels (ideally measures of the effector components of the immune system, such as antibody or T-cell concentrations) throughout the course of infection to determine the most appropriate model (similar approaches have been used in free-living ecology 
to quantify the predator's functional response; Carpenter et al. 1994; Harrison, 1995; Jost and Arditi, 2001). However, while these approaches, if logistically possible, may allow Type 1 functional responses to be separated from other types, selecting between Types 2 and 3 may be more difficult. The key component of a Type 3 functional response is the initial non-linearity at low prey (parasite) densities, and high quality data at these low densities are required to achieve sufficient resolution to differentiate between these responses (Otterstatter and Thomson, 2006). Typically, however, the inocula given in experimental trials tend to be very large, to ensure successful infection, and are often constant between experiments, giving little information on parasite clearance across a sufficient range of densities. Hence, even though sophisticated modelling and statistical techniques may provide some insight into approximate parameter values (e.g., Riley et al. 2003), it is unlikely to be possible to accurately determine the functional response without gathering data at these low pathogen densities. Given the importance of determining the shape of the functional response in terms of the dynamics of the parasite community, more detailed quantitative studies, especially of low-dose infections are urgently required.

Throughout this paper we have assumed that any immunological impact of one parasite species is inevitably felt by another co-infecting parasite species, regardless of their site of infection. Effectively, this assumes that such immune responses are systemic and occur throughout the host. However, immune responses are typically compartmentalized, such that their effects are localized to the site of infection, and there may be little interaction between responses mounted towards co-infecting parasites that occur in different regions of the host (Lamb et al. 2005). Therefore, spatial heterogeneity in the site of infection may reduce the impact of potential immunemediated interspecific parasite interactions.

A further limitation of the present models is that, although we have concentrated on parasite interactions mediated by the host's immune response, there may be other powerful mechanisms of interaction acting within the parasite community. In particular, parasites which infect in close proximity to each other may experience high levels of interspecific competition, affecting their survival and/or fecundity, either through resource competition, the release of chemical mediators, or physical constraints within the host (Keymer, 1982; Christensen et al. 1987; Behnke, 2008). Classic examples of such interspecific competition occur within gut helminths, where the presence of co-infecting parasite species has been shown to affect the physical distribution and size of each other, relative to their distribution in single infections (Holmes, 1961, 1962). However, such direct competitive effects may be more widespread than previously thought; resourcemediated interspecific competition has been shown to occur between anaemia-causing macroparasites and microparasites that depend on red blood cells (Graham, 2008). Clearly, there is a need to develop appropriate theoretical frameworks of such direct interactions, based initially on classic ecological models of interspecific competition (Volterra, 1926; Gause, 1934) but extended to consider, for example, potential non-linear relationships between competitive ability and parasite load, and also the interaction between such 'bottom-up' effects acting concurrently with the 'top-down' immunological effects considered here (Graham, 2008).

Finally, it should be noted that although there are a number of similarities between free-living ecological communities and within-host parasite communities, there are also several key differences. One important difference is that immune responses that target helminths may not result in parasite mortality, but frequently affect parasite fecundity (Tompkins and Hudson, 1999). In this case the current composition of the parasite community within the focal host individual is unlikely to be affected by the immune response; such immunity will reduce the transmission of parasites between hosts by reducing output to the environment rather than directly affecting the current abundance of each parasite species within the host. Furthermore, a number of parasites, both micro- and macroparasites, can avoid, suppress or divert the host's immune response. For example, HIV is able to evade the immune response and even consumes components of it (CD4 cells). Furthermore, immune system dynamics may not be well characterized by predator-prey interactions, where factors such as time delays and 'programtype' immune stimulation (Antia et al. 2003) may alter the relationship between pathogen and immune response levels (Fenton et al. 2006; Wodarz, 2006). Clearly these processes fall outside those usually experienced in free-living community ecology. Nevertheless, adopting a community ecology approach to studying within-host parasite communities will provide a valuable starting point for understanding the broad dynamics of many co-infecting parasites, allowing insight into how and why certain infections persist, others are eliminated, and others overwhelm the immune response and kill the host.

\section{ACKNOWLEDGMENTS}

The authors would like to thank two anonymous reviewers and Beth Vigliotti at Penn State University for their very helpful comments. A. F. was funded by a Fellowship from NERC.

REFERENCES

Abbas, A. K., Murphy, K. M. and Sher, A. (1996).

Functional diversity of helper T lymphocytes. Nature, London 383, 787-793. 
Antia, R., Bergstrom, C. T., Pilyugin, S. S., Kaech, S. M. and Ahmed, R. (2003). Models of CD8+ responses: 1 . What is the antigen-independent proliferation program. Fournal of Theoretical Biology 221, 585-598.

Antia, R. and Koella, J. (2004). Theoretical immunology - Parasitic turncoat. Nature, London 429, 511-513

Antia, R., Koella, J. C. and Perrot, V. (1996). Models of the within-host dynamics of persistent mycobacterial infections. Proceedings of the Royal Society of London, $B$ 263, 257-263.

Antia, R. and Lipsitch, M. (1997). Mathematical models of parasite responses to host immune defences. Parasitology 115, S155-S167.

Bassetti, S., Bischoff, W. E. and Sherertz, R. J. (2005). Are SARS superspreaders cloud adults? Emerging Infectious Diseases 11, 637-638.

Behnke, J. M. (2008). Structure in parasite component communities in wild rodents: predictability, stability, associations and interactions ... or pure randomness? Parasitology 135, 751-766.

Ben-Smith, A., Wahid, F. N., Lammas, D. A. and Behnke, J. M. (1999). The relationship between circulating and intestinal Heligmosomoides polygyrus-specific IgG(1) and IgA and resistance to primary infection. Parasite Immunology 21, 383-395.

Bentwich, Z., Kalinkovich, A., Weisman, Z., Borkow, G., Beyers, N. and Beyers, A. D. (1999). Can eradication of helminthic infections change the face of AIDS and tuberculosis? Immunology Today 20, 485-487.

Bonsall, M. B. and Hassell, M. P. (1997). Apparent competition structures ecological assemblages. Nature, London 388, 371-373.

Borer, E. T., Anderson, K., Blanchette, C. A., Broitman, B., Cooper, S. D., Halpern, B. S., Seabloom, E. W. and Shurin, J. B. (2002). Topological approaches to food web analyses: a few modifications may improve our insights. Oikos 99, 397-401.

Bottomley, C., Isham, V. and Basanez, M. G. (2007). Population biology of multispecies helminth infection: competition and coexistence. Fournal of Theoretical Biology 244, 81-95.

Brady, M. T., O’Neill, S. M., Dalton, J. P. and Mills, K. H. G. (1999). Fasciola hepatica suppresses a protective Th1 response against Bordetella pertussis. Infection and Immunity 67, 5372-5378.

Buric, N., Mudrinic, M. and Vasovic, N. (2001). Time delay in a basic model of the immune response. Chaos Solitons \& Fractals 12, 483-489.

Callard, R. E. and Yates, A. J. (2005). Immunology and mathematics: crossing the divide. Immunology $\mathbf{1 1 5}$, 21-33.

Carpenter, S. R., Cottingham, K. L. and Stow, C. A. (1994). Fitting predator-prey models to time series with observation errors. Ecology 75, 1254-1264.

Christensen, N. O., Nansen, P., Fagbemi, B. O. and Monrad, J. (1987). Heterologous antagonistic and synergistic interactions between helminths and between helminths and protozoans in concurrent experimental infection of mammalian hosts. Parasitology Research 73, $387-410$.
Cox, F. E. G. (2001). Concomitant infections, parasites and immune responses. Parasitology 122, S23-S38.

Ebert, D. (1994). Virulence and local adaptation of a horizontally transmitted parasite. Science $\mathbf{2 6 5}$, 1084-1086.

Fenton, A. (2008). Worms and germs: the population dynamic consequences of microparasite-macroparasite co-infection. Parasitology 135, 1545-1560.

Fenton, A., Lamb, T. and Graham, A. L. (2008). Optimality analysis of Th1/Th2 immune responses during microparasite-macroparasite co-infection, with epidemiological feedbacks. Parasitology 135, 841-853.

Fenton, A., Lello, J. and Bonsall, M. B. (2006). Pathogen responses to host immunity: the impact of time delays and memory on the evolution of virulence. Proceedings of the Royal Society of London B 273, 2083-2090.

Ferguson, N. M., Galvani, A. P. and Bush, R. M. (2003). Ecological and immunological determinants of influenza evolution. Nature, London 422, 428-433.

Gause, G. F. (1934). The Struggle for Existence. Williams and Wilkins, Baltimore, MD, USA.

Gause, G. F. (1935). Experimental demonstration of Volterra's periodic oscillations in the numbers of animals. Fournal of Experimental Biology 12, 44-48.

Gause, G. F. (1936). Further studies of interaction between predators and prey. The Fournal of Animal Ecology 5, 1-18.

Gog, J. R. and Grenfell, B. T. (2002). Dynamics and selection of many-strain pathogens. Proceedings of the National Academy of Sciences, USA 99, 17209-17214.

Graham, A. L. (2008). Ecological rules governing helminth-microparasite coinfection. Proceedings of the National Academy of Sciences, USA 105, 566-570.

Gupta, S., Swinton, J. and Anderson, R. M. (1994). Theoretical studies of the effects of heterogeneity in the parasite population on the transmission dynamics of malaria. Proceedings of the Royal Society of London, $B$ 256, 231-238.

Harrison, G. W. (1995). Comparing predator-prey models to Luckinbill's experiment with Didinium and Paramecium. Ecology 76, 357-374.

Holmes, J. C. (1961). Effects of concurrent infections on Hymenolepis diminuta (Cestoda) and Moniliformis dubius (Acanthocephala). 1. General effects and comparison with crowding. Fournal of Parasitology 47, 209-216.

Holmes, J. C. (1962). Effects of concurrent infections on Hymenolepis diminuta (Cestoda) and Moniliformis dubius (Acanthocephala). Effects on growth. Fournal of Parasitology 48, 87-96.

Holt, R. D. (1977). Predation, apparent competition, and structure of prey communities. Theoretical Population Biology 12, 197-229.

Holt, R. D. (1983). Optimal foraging and the form of the predator isocline. American Naturalist 122, 521-541.

Holt, R. D. and Dobson, A. P. (2006). Extending the principles of community ecology to address the epidemiology of host-pathogen systems. In Disease Ecology: Community Structure and Pathogen Dynamics (ed. Collinge, S. K. and Ray, C.), pp. 6-27. Oxford University Press, Oxford, UK. 
Holt, R. D. and Lawton, J. H. (1994). The ecological consequences of shared natural enemies. Annual Review of Ecology and Systematics 25, 495-520.

Jost, C. and Arditi, R. (2001). From pattern to process: identifying predator-prey models from time-series data. Population Ecology 43, 229-243.

Keymer, A. (1982). Density-dependent mechanisms in the regulation of intestinal helminth populations. Parasitology 84, 573-587.

Koelle, K., Rodo, X., Pascual, M., Yunus, M. and Mostafa, G. (2005). Refractory periods and climate forcing in cholera dynamics. Nature, London 436, 696-700.

Lamb, T. J., Graham, A. L., Le Goff, L. and Allen, J. E. (2005). Co-infected C57BL/6 mice mount appropriately polarized and compartmentalized cytokine responses to Litomosoides sigmodontis and Leishmania major but disease progression is altered. Parasite Immunology 27, 317-324.

Lello, J., Boag, B., Fenton, A., Stevenson, I. R. and Hudson, P. J. (2004). Competition and mutualism among the gut helminths of a mammalian host. Nature, London 428, 840-844.

Liesenfeld, O., Dunay, I. R. and Erb, K. J. (2004). Infection with Toxoplasma gondii reduces established and developing $\mathrm{Th} 2$ responses induced by Nippostrongylus brasiliensis infection. Infection and Immunity 72, 3812-3822.

Marshall, B. G., Mitchell, D. M., Shaw, R. J., Marais, F., Watkins, R. M. and Coker, R. J. (1999). HIV and tuberculosis co-infection in an inner London hospital - a prospective anonymized seroprevalence study. Fournal of Infection 38, 162-166.

May, R. M. (1974). Stability and Complexity in Model Ecosystems. Princeton University Press, Princeton, NJ, USA.

Murdoch, W. W., Briggs, C. J. and Nisbet, R. M. (2003). Consumer-Resource Dynamics. Princeton University Press, Princeton, NJ, USA.

Nowak, M. A. and May, R. M. (2000). Virus Dynamics: Mathematical Principles of Immunology and Virology. Oxford University Press, Oxford, UK.
Otterstatter, M. C. and Thomson, J. D. (2006).

Within-host dynamics of an intestinal pathogen of bumble bees. Parasitology 133, 749-761.

Pedersen, A. B. and Fenton, A. (2007). Emphasising the ecology in parasite community ecology. Trends in Ecology E Evolution 22, 133-139.

Perelson, A. S. (2002). Modelling viral and immune system dynamics. Nature Reviews Immunology 2, 28-36.

Pilyugin, S. S. and Antia, R. (2000). Modeling immune responses with handling time. Bulletin of Mathematical Biology 62, 869-890.

Read, A. F., Graham, A. L. and Raberg, L. (2008). Animal defenses against infectious agents: is damage control more important than pathogen control? PLoS Biology 6, 2638-2641.

Riley, S., Donnelly, C. A. and Ferguson, N. M. (2003). Robust parameter estimation techniques for stochastic within-host macroparasite models. Fournal of Theoretical Biology 225, 419-430.

Rohani, P., Green, C. J., Mantilla-Beniers, N. B. and Grenfell, B. T. (2003). Ecological interference between fatal diseases. Nature, London 422, 885-888.

Shen, Z., Ning, F., Zhou, W. G., He, X., Lin, C. Y., Chin, D. P., Zhu, Z. H. and Schuchat, A. (2004). Superspreading SARS events, Beijing, 2003. Emerging Infectious Diseases 10, 256-260.

Sole, R. V. and Montoya, J. M. (2001). Complexity and fragility in ecological networks. Proceedings of the Royal Society of London, B 268, 2039-2045.

Tompkins, D. M. and Hudson, P. J. (1999). Regulation of nematode fecundity in the ring-necked pheasant (Phasianus colchicus): not just density dependence. Parasitology 118, 417-423.

Volterra, V. (1926). Fluctuations in the abundance of a species considered mathematically. Nature, London 118, 558-560.

Wodarz, D. (2006). Ecological and evolutionary principles in immunology. Ecology Letters 9, 694-705.

Zinkernagel, R. M. (1996). Immunology taught by viruses. Science 271, 173-178. 